\title{
A spiking recurrent neural network with phase change memory synapses for decision making
}

\author{
G. Pedretti ${ }^{1 *}$, V. Milo ${ }^{1}$, S. Hashemkhani ${ }^{1}$, P. Mannocci $^{1}$, O. Melnic ${ }^{1}$, E. Chicca ${ }^{2}$, and D. Ielmini ${ }^{1}$ \\ ${ }^{1}$ Dipartimento di Elettronica, Informazione e Bioingegneria, Politecnico di Milano and IU.NET, Milano 20133, Italy \\ ${ }^{2}$ Faculty of Technology and Cognitive Interaction Technology Center of Excellence (CITEC), Bielefeld University, \\ Bielefeld, Germany *email: giacomo.pedretti@ polimi.it
}

\begin{abstract}
Neuronal activity of recurrent neural networks (RNNs) experimentally observed in the hippocampus is widely believed to play a key role for mammalian ability to associate concepts and make decisions. For this reason, RNNs have rapidly gained strong interest as computational enabler of brain-inspired cognitive functions in hardware. From the technology viewpoint, nonvolatile memory devices such as phase change memory (PCM) and resistive switching memory (RRAM) have become a key asset to allow for high synaptic density and biorealistic cognitive functionality. In this work, we demonstrate for the first time associative learning and decision making in a hardware Hopfield RNN with 6 spiking neurons and PCM synapses via storage, recall and competition of attractor states. We also experimentally demonstrate the solution of a constraint satisfaction problem (CSP) namely a Sudoku with size $2 \times 2$ in hardware and 9x9 in simulation. These results support spiking RNNs with PCM devices for the implementation of decision making capabilities in hardware neuromorphic systems.
\end{abstract}

Keywords_phase change memory (PCM); Hebbian learning; spiking recurrent neural network (RNN); associative memory; decision making; constraint satisfaction problems (CSPs).

\section{INTRODUCTION}

Over millions of years, mammalian brain evolution has been driven by the exponential progress of the computing capabilities resulting in fundamental human cognitive primitives such as reasoning, learning, classification, adaptation and decision making. To achieve such a deep, multifaceted computing performance, the human brain processes sensory information via dense neural networks of spiking neurons and adaptable synapses which change their weights according to biological plasticity rules. Many neurophysiological studies suggest that neuronal activity in recurrent neural networks (RNNs) within the hippocampus may play a crucial role for developing key human abilities such as associative memory and contextdependent decision making [1], [2]. These experiments have spurred the intense investigation of the computational power of RNNs via accurate mathematical models [3], [4] supporting the development of spiking RNN prototypes in complementary metal-oxide semiconductor (CMOS) technology [5]. Since

This article has received funding from the European Research Council (ERC) under the European Union's Horizon 2020 research and innovation programme (grant agreement No. 648635) and the Cluster of Excellence Cognitive Interaction Technology "CITEC" (EXC 277) at Bielefeld University, which is funded by the German Research Foundation (DFG).

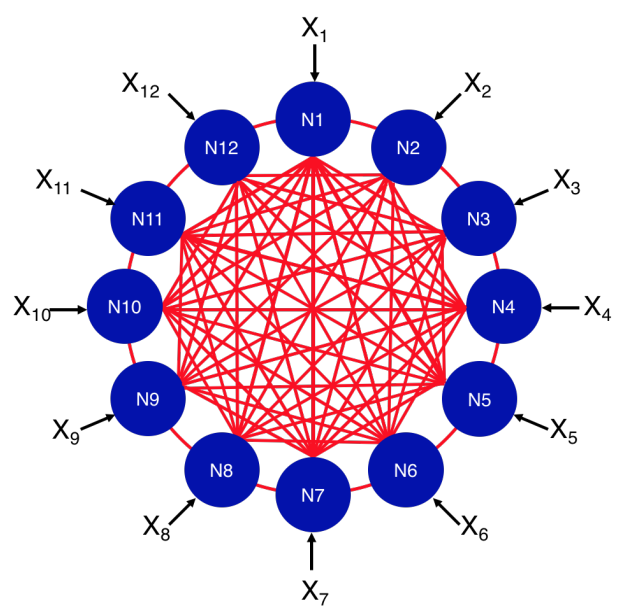

Fig. 1. Sketch of a Hopfield recurrent neural network (RNN). Each neuron (blue) is connected to other neurons via all-to-all bidirectional synaptic connections (red). Neuron self-connection is not allowed in Hopfield RNNs.

then, CMOS-based spiking RNNs have been shown to enable neuromorphic tasks such as associative memory [6], [7], pattern completion [7] and decision making [8], [9], also exhibiting a great potential as solver of constraint satisfaction problems (CSPs) [10] including Sudoku [11].

Although the adoption of CMOS technology has allowed to achieve significant milestones in this field, novel materialbased memory devices such as the resistive switching memory (RRAM) and phase change memory (PCM) have gained increasing interest to bridge the gap in terms of energy efficiency and synaptic density with biological neural system thanks to their non-volatile storage, scalability, and low power consumption [12], [13]. Based on these memory technologies, hardware demonstrations [14], [15] and simulation studies [16]-[19] of spiking RNNs have been recently reported.

In this paper, we present a hardware Hopfield RNN with 6 spiking neurons and 30 PCM synapses capable of learning and recalling attractor memory states using a Hebbian-type weight update rule. The competition between attractor states is investigated in experiments and simulations to demonstrate RNN ability to implement decision making. Finally, a $2 \times 2$ Sudoku is solved in hardware and a 9x9 Sudoku is simulated 


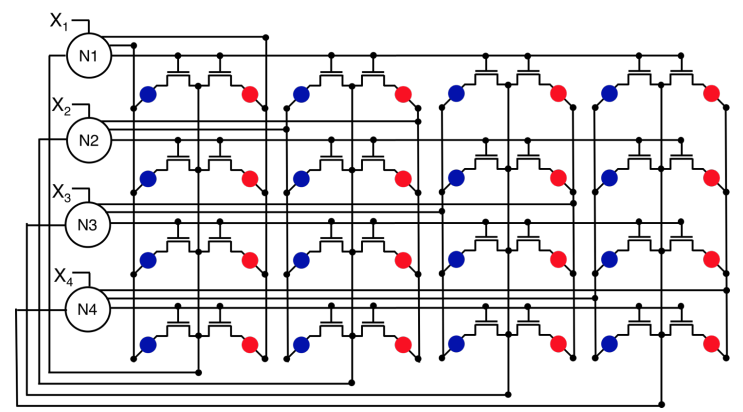

Fig. 2. Circuit schematic of the implemented Hopfield RNN. Synapses consist of 1T1R PCM devices arranged in a parallel configuration with excitatory (blue) and inhibitory (red) paths. The gates and top electrodes are connected to the neuron rows and columns, respectively. External stimulation causes neuron fires, which in turn lead to spiking currents flowing back to neuron for competition/cooperation dynamics within the attractors.

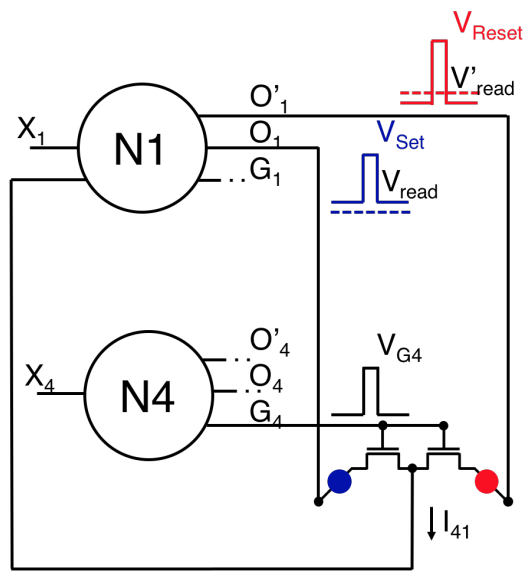

Fig. 3. Hebbian learning. Fire of neurons $\mathrm{N}_{1}$ and $\mathrm{N}_{4}$ results in high-voltage overlapping spikes at $\mathrm{G}_{4}, \mathrm{O}_{1}$ and $\mathrm{O}_{1}$, thus leading to the potentiation of excitatory synapses and the depression of inhibitory synapses. As a result, neurons that fire together wire together according to the Hebb's postulate.

via our Monte Carlo (MC) model of spiking RNN.

\section{HOPFIELD RNN WITH PCM SYNAPSES}

Hopfield RNN is a type of RNN providing a fundamental computational substrate to implement key human primitives such as associative memory [3]. Fig. 1 shows the schematic illustration of a Hopfield RNN consisting of $\mathrm{N}$ neurons where each neuron receives an external stimulation input $\mathrm{X}_{i}$ $(i=1: N)$ and is connected to all the other $\mathrm{N}-1$ neurons via synaptic connections. To implement the Hopfield RNN in hardware, we developed a circuit like the one illustrated in Fig. 2, which contains only $\mathrm{N}=4$ neurons for the sake of simplicity [17]. In this RNN architecture, each synapse consists of 2 PCM cells with one-transistor/one-resistor (1T1R) structure to implement the excitatory and inhibitory weights, respectively, while each neuron has 3 output terminals, namely (i) the transistor gates along the row, (ii) the top electrodes of excitatory PCM devices along the column and (iii) the top electrodes of inhibitory PCM devices along the column, (a)
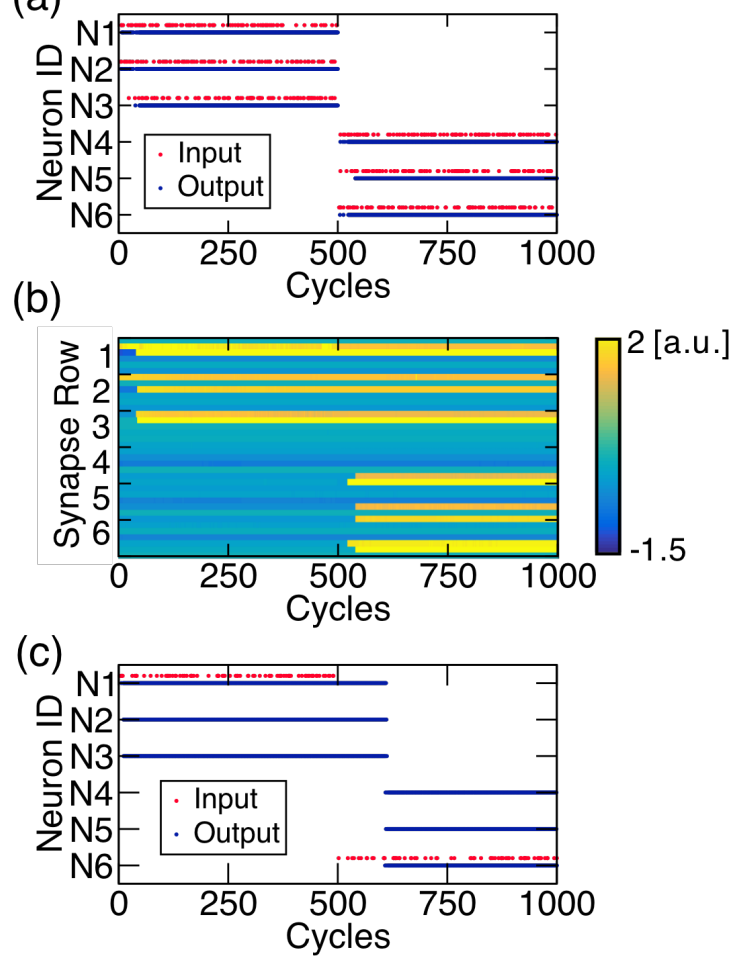

Fig. 4. Hardware demonstration of learning of two attractors. (a) First, $\mathrm{N}_{1}, \mathrm{~N}_{2}, \mathrm{~N}_{3}$ are stimulated for 500 cycles, followed by stimulation of $\mathrm{N}_{4}$, $\mathrm{N}_{5}, \mathrm{~N}_{6}$ for the following 500 cycles. Simultaneous stimulation of neurons causes synaptic potentiation, thus the formation of attractors. (b) Measured evolution of synaptic conductances during the stimulation of (a), evidencing the potentiation of synapses within the first and second attractor, which is responsible for cooperation and associative learning. Non-stimulated synapses remain with low weight, which is responsible for attractor competition. (c) Hardware demonstration of the attractor recall. Stimulation of $\mathrm{N}_{1}$ alone causes the recall of Attractor \#1. Stimulation of $\mathrm{N}_{6}$ leads to the recall of Attractor \#2, while the Attractor \#1 is switched off by inhibitory synapses.

which are respectively controlled by signals $\mathrm{G}, \mathrm{O}$, and $\mathrm{O}^{\prime}$, as shown in Fig. 3. Note that $\mathrm{O}$ and $\mathrm{O}^{\prime}$ are biased by positive and negative read voltages, respectively, to enable positive/negative currents across excitatory/inhibitory synapses. Considering the interacting neurons $\mathrm{N}_{1}$ and $\mathrm{N}_{4}$, Fig. 3 shows that the Hopfield RNN can implement Hebbian learning according to the rule "neurons that fire together, wire together". In fact, as the neurons fire at the same time, then a high signal is delivered at both $\mathrm{G}_{4}$ and top electrode $\mathrm{O}_{1}$ causing the potentiation of the excitatory synapse by the application of a voltage $\mathrm{V}_{\text {Set }}$ to the corresponding PCM which results in a material crystallization and a consequent transition to the low resistance state (LRS). At the same time, the spike to the top electrode $\mathrm{O}_{1}$ ' causes the depression of the inhibitory synapse by the application of a voltage $\mathrm{V}_{\text {Reset }}$ to the corresponding PCM which results in a material amorphization and a consequent transition to the high resistance state (HRS). Also, at the same time, the spiking of terminals $\mathrm{G}_{1}, \mathrm{O}_{4}$ and $\mathrm{O}_{4}$ ' causes the learning (potentiation of excitatory synapse and depression of inhibitory synapse) at the symmetric position $(4,1)$. As a result, this scheme allows for 

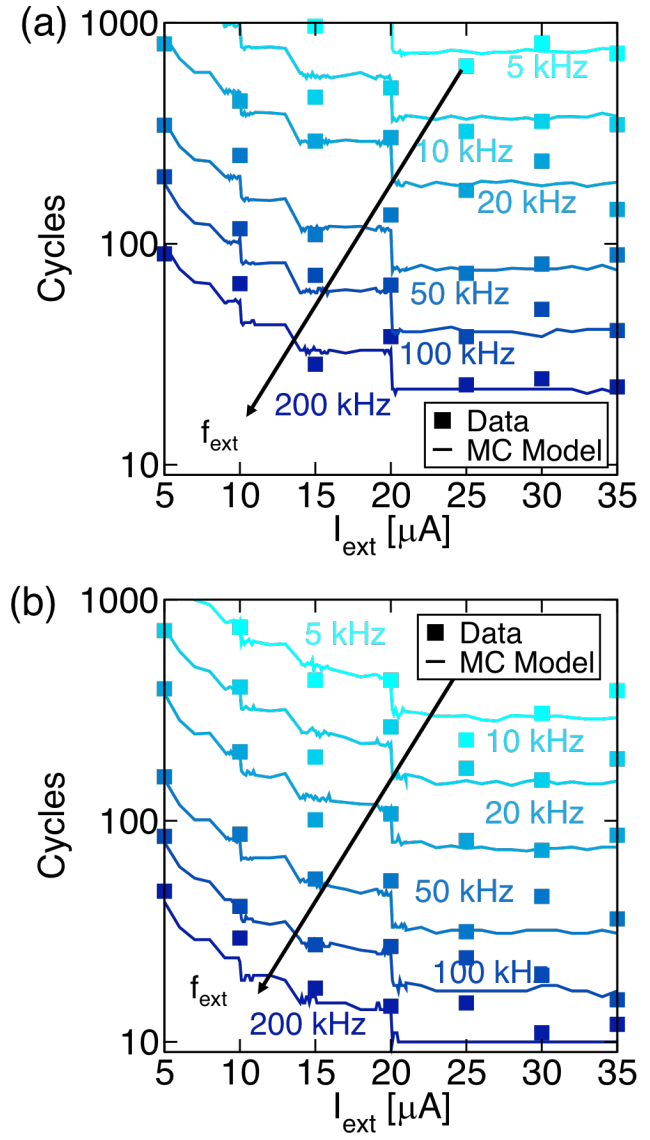

Fig. 5. Number of cycles to recall a stored attractor as a function of the stimulating current $\mathrm{I}_{\text {ext }}$ and frequency $\mathrm{f}_{\text {ext }}$ for (a) one stimulated neuron and (b) two stimulated neurons. The recall time decreases for increasing $\mathrm{I}_{\text {ext }}$ and increasing $\mathrm{f}_{\text {ext }}$. The abrupt steps at $\mathrm{I}_{\text {ext }}=20 \mu \mathrm{A}$ are due to the decrease of the number of spikes needed to reach the threshold from two to one.

a symmetric adaptation of the synaptic weights where $\mathrm{W}_{i j}=$ $\mathrm{W}_{j i}$, with $i \neq j$, to achieve the storage of stable attractor states [3].

\section{AsSOCIATIVE MEMORY IN HOPFIELD RNN}

Based on Hebbian learning scheme, we demonstrated the learning of two attractor states in a hardware Hopfield RNN with 6 spiking neurons. This experiment was carried out by applying an external stimulation to neurons $\left(\mathrm{N}_{1}, \mathrm{~N}_{2}\right.$, $\mathrm{N}_{3}$ ) for 500 cycles via high-frequency Poisson spike trains followed by external stimulation to neurons $\left(\mathrm{N}_{4}, \mathrm{~N}_{5}, \mathrm{~N}_{6}\right)$ for the subsequent 500 cycles (Fig. 4a). As a result, the stochastic coactivation of neurons within each pool caused the potentiation of the respective excitatory synapses, thus leading to the formation of the corresponding attractor states, namely Attractor \#1 of neurons $\mathrm{N}_{1}, \mathrm{~N}_{2}$, and $\mathrm{N}_{3}$, and Attractor \#2 of neurons $\mathrm{N}_{4}, \mathrm{~N}_{5}$, and $\mathrm{N}_{6}$ as shown in Fig. $4 \mathrm{~b}$.

After the learning phase, we experimentally demonstrated the recall process of the 2 attractors, where a whole attractor is activated by the stimulation of just one or few of its neurons. Fig. 4c shows that the external stimulation of a single neuron $\left(\mathrm{N}_{1}\right)$ within Attractor \#1 enables the recall of the whole

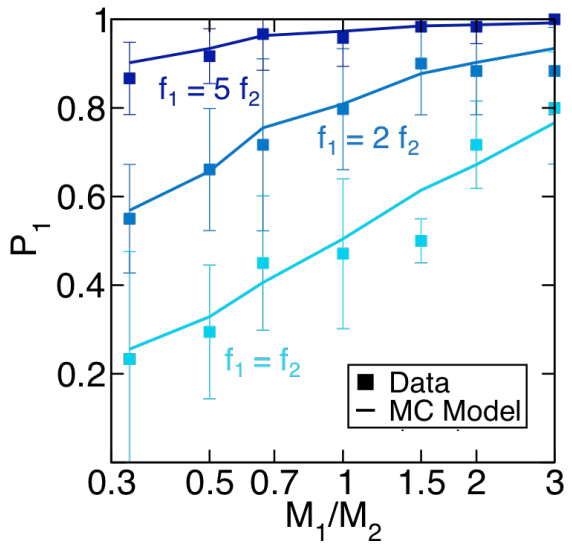

Fig. 6. Probability to recall Attractor \#1 as a function of the ratio between the number of stimulated neurons within Attractor \#1 $\left(\mathrm{M}_{1}\right)$ and the number of stimulated neurons within Attractor \#2 $\left(\mathrm{M}_{2}\right)$ for increasing ratio of corresponding stimulation frequencies $\mathrm{f}_{1}$ and $\mathrm{f}_{2} . \mathrm{P}_{1}$ increases with $\mathrm{M}_{1} / \mathrm{M}_{2}$ and with $\mathrm{f}_{1} / \mathrm{f}_{2}$, due to the stronger stimulation of Attractor \#1.

Attractor \#1 resulting in the firing activity of all neurons within the first attractor, which remain active even after the external stimulation has been discontinued. The external stimulation of a single neuron $\left(\mathrm{N}_{6}\right)$ within Attractor \#2 leads to the activation of Attractor \#2 and the deactivation of Attractor \#1 thanks to the inhibitory synapses that connect neurons in the first attractor to those in the second attractor. Note that the attractor recall forms the basis for the associative memory, where the activation of a sensory input (e.g., the bell ringing in the case of Pavlov's dog) results in the activation of a linked memory (e.g., food).

Hopfield RNN operation to achieve memory retrieval was extensively investigated performing both experiments and simulations via a MC model. Fig. 5a shows the number of cycles to recall an attractor state as a function of the amplitude of the external spikes $\mathrm{I}_{\text {ext }}$ and their frequency $\mathrm{f}_{\text {ext }}$ of stimulating one neuron in the attractor. The results show that the time to recall an attractor state decreases for increasing $\mathrm{f}_{\text {ext }}$ and for increasing $\mathrm{I}_{\text {ext }}$. The occurrence of abrupt steps is due to $\mathrm{I}_{\text {ext }}$ becoming equal to submultiples of the threshold current $\mathrm{I}_{t h}=40 \mu \mathrm{A}$ and marks the transition to a lower number of external spikes needed to hit the threshold. Fig. 5b shows similar results for the simultaneous stimulation of two neurons in the same attractor, indicating that the stimulation of two neurons accelerates the recall process and allows for a lower number of cycles for the same $\mathrm{f}_{\text {ext }}$ with respect to singleneuron stimulation. This is the result of doubling the input excitation current to reactivate a certain attractor, which thus requires less time to hit the threshold.

\section{DECISION MAKING IN HOPFIELD RNN}

Besides associative memory, extensive studies have shown that RNNs can also reproduce another fundamental biological primitive namely decision making [20]. The ability to make decisions was validated in our RNN by stochastic stimulation of two competitive attractors as evidenced in Fig. 6. Here, the 
(a)

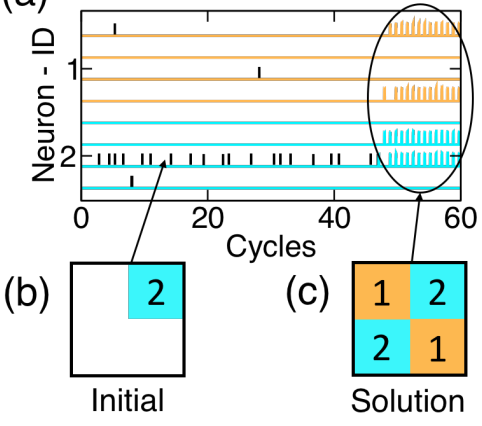

Fig. 7. Hardware solution of a $2 \times 2$ Sudoku puzzle. (a) Stimulation and internal neuron spikes during the solution of the Sudoku. (b) Stimulation of clue '2' in position $(1,2)$ combined with random noise stimulation of other neurons results in the activation of the solution $12 ; 21$ after about 50 spikes (c).

probability $\mathrm{P}_{1}$ to recall Attractor \#1 is reported as a function of the ratio $M_{1} / M_{2}$, where $M_{1}$ and $M_{2}$ indicate the number of stimulated neurons within Attractor \#1 and Attractor \#2, respectively, for variable ratio $\mathrm{f}_{1} / \mathrm{f}_{2}$ of stimulating frequencies of two attractor states, namely $\mathrm{f}_{1} / \mathrm{f}_{2}=1,2$ or 5 . Experimental results show that $P_{1}$ increases with increasing $M_{1} / M_{2}$ and $\mathrm{f}_{1} / \mathrm{f}_{2}$, which is supported by the good agreement between the simulations of our MC model and the experimental data. These results indicate that context-dependent probabilistic decision making enriches the portfolio of biological behaviors achievable in hardware using PCM-based Hopfield RNN circuits.

\section{Solution of a Sudoku PUZZLE IN Hopfield RNN}

Sudoku is a typical CSP where the use of Hopfield RNNs can provide a fast and efficient tool for the solution. In fact, a Hopfield RNN allows to iteratively achieve the CSP solution by minimizing the cost function $E=-1 / 2 \sum_{i j} W_{i j} V_{i} V_{j}$ where $\mathrm{V}_{i}$ is the voltage applied to the external stimulation of neuron $\mathrm{N}_{i}$, and $\mathrm{W}_{i j}$ is the net conductance of the synapses connecting neuron $i$ and neuron $j$, obtained as the excitatory conductance minus the inhibitory conductance. The cost function is minimized by simulated annealing [21], which is based on the use of random noise to escape from local minima to the global minimum representing the right solution of the CSP problem [22]-[24]. Specifically, a Sudoku consists of a NxN puzzle where each of the $\mathrm{N}$ digits $(1,2,3,4,5,6,7,8,9)$ must appear only once in the same column, row, or sub-square. Sudoku can be implemented in a Hopfield RNN with $\mathrm{N}^{3}$ neurons mapping constraints/initial conditions via excitatory synapses (e.g., a '1' in a certain position excites the other digits on the same row/column) and inhibitory synapses (e.g., a '1' in a certain position inhibits ' 1 ' on the same row/column and the other digits in the same position).

To support the ability of Hopfield RNNs to solve Sudoku, we demonstrated the solution of a $2 \times 2$ Sudoku in a hardware Hopfield RNN with 8 software stochastic neurons and an $8 \times 8$ array of 1T1R PCM synapses. As evidenced by the raster plot in Fig. 7a, the RNN initially receives low frequency random noise spikes along with high frequency spikes to (a)

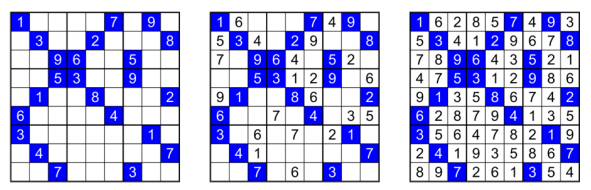

(b)

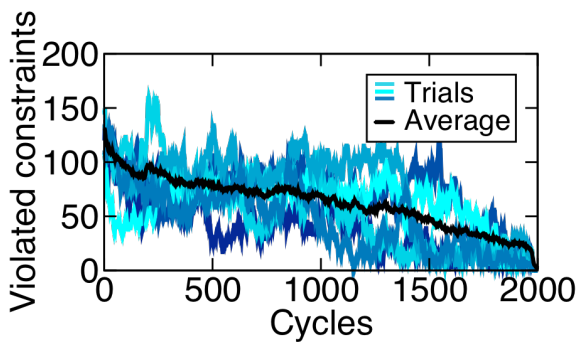

Fig. 8. Monte Carlo (MC) simulations of a 9x9 Sudoku. (a) Clues of the Escargot puzzle, intermediate solution after 1000 cycles, and final solution of 9x9 Sudoku. (b) Number of violated constraints as a function of cycles for 10 trials of the MC simulations.

implement the 2x2 Sudoku initial condition shown in Fig. $7 \mathrm{~b}$. After about 50 cycles, the external stimulation combined with random noise causes the neurons of RNN to emit output spikes corresponding to the Sudoku solution shown in Fig. 7c. Finally, we scaled up the problem complexity by solving a hard 9x9 Sudoku, called Escargot puzzle, by a calculated Hopfield RNN with 729 neurons and 729x729 synapses. Fig. 8a shows (left) the initial hints, (center) the intermediate state calculated after 1000 cycles, and (right) the final state reached after 2000 cycles corresponding to Sudoku solution. To support the computational power of our RNN, Fig. $8 \mathrm{~b}$ shows the number of violated constraints as a function of cycles for 10 trials of the $\mathrm{MC}$ simulations evidencing that computational error rapidly decreases reaching zero within 2000 cycles. These results support our PCM-based Hopfield RNN as a fast and computationally efficient solver of hard CSPs.

\section{CONCLUSIONS}

We present a novel hardware Hopfield RNN with spiking neurons and excitatory/inhibitory synapses based on PCM devices capable of implementing biologically inspired functions as associative memory and decision making. Furthermore, we used our spiking RNN as solver of CSPs demonstrating a 2x2 Sudoku in hardware and a very hard 9x9 Sudoku in simulation. The experimental/simulation results support our PCM-based Hopfield RNN as a significant tool for efficiently solving a wide range of computationally expensive tasks.

\section{REFERENCES}

[1] Y. Miyashita,"Neuronal correlate of visual associative long-term memory in the primate temporal cortex," Nature, vol. 335, no. 6193, pp. 817-820, 1988, DOI: $10.1038 / 335817 \mathrm{a} 0$.

[2] Y. Miyashita, E. T. Rolls, P. M. B. Cahusac, H. Niki and J. D. Feigenbaum, "Activity of hippocampal formation neurons in the monkey related to a conditional spatial response task," Journal of Neurophysiology, vol. 61, no. 3, pp. 669-678, 1989, DOI: 10.1152/jn.1989.61.3.669.

[3] J. J. Hopfield, "Neural networks and physical systems with emergent collective computational abilities," Proc. Natl. Acad. Sci. USA (PNAS), vol. 79, no. 8, pp. 2554-2558, 1982, DOI: 10.1073/pnas.79.8.2554. 
[4] D. J. Amit, "Modeling brain function-The world of attractor neural networks," Cambridge University Press, Cambridge, 1989.

[5] E. Chicca, D. Badoni, V. Dante, M. D'Andreagiovanni, G. Salina, L. Carota, S. Fusi, and P. Del Giudice, "A VLSI recurrent network of integrate-and-fire neurons connected by plastic synapses with long-term memory," IEEE Trans. Neural Netw., vol. 14, no. 5, pp. 1297-1307, 2003, DOI: $10.1109 / \mathrm{TNN} .2003 .816367$.

[6] G. Indiveri and S.-C. Liu, "Memory and information processing in neuromorphic systems," Proc. IEEE, vol. 103, no. 8, pp. 1379-1397, 2015, DOI: 10.1109/JPROC.2015.2444094.

[7] M. Giulioni, F. Corradi, V. Dante and P. Del Giudice, "Real time unsupervised learning of visual stimuli in neuromorphic VLSI systems," Sci. Rep., vol. 5, no. 14730, 2015, DOI: 10.1038/srep14730.

[8] F. Corradi, H. You, M. Giulioni and G. Indiveri, "Decision making and perceptual bistability in spike-based neuromorphic VLSI systems," in IEEE Int. Symp. Circuits and Systems (ISCAS), pp. 2708-2711, 2015, DOI: 10.1109/ISCAS.2015.7169245.

[9] D. Liang and G. Indiveri, "A neuromorphic computational primitive for robust context-dependent decision making and context-dependent stochastic computation," IEEE Trans. on Circuits and Systems II: Express Briefs, vol. 66, no. 5, pp. 843-847, 2019, DOI: 10.1109/TCSII.2019.2907848.

[10] H. Mostafa, L. K. Müller and G. Indiveri, "An event-based architecture for solving constraint satisfaction problems," Nat. Commun., vol. 6, no. 8941, 2015, DOI: 10.1038/ncomms9941.

[11] J. Binas, G. Indiveri, and M. Pfiffer, "Spiking analog VLSI neuron assemblies as constraint satisfaction problem solvers," in IEEE Int. Symp. Circuits and Systems (ISCAS), pp. 2094-2097, 2016, DOI: 10.1109/ISCAS.2016.7538992.

[12] D. Kuzum, S. Yu, and H.-S. P. Wong, "Synaptic electronics: materials, devices and applications," Nanotechnology, vol. 24, no. 382001, 2013, DOI: $10.1088 / 0957-4484 / 24 / 38 / 382001$.

[13] D. Ielmini and S. Ambrogio, "Emerging neuromorphic devices," Nanotechnology 31 (9), p. 092001, 2019, DOI: 10.1088/1361-6528/ab554b.

[14] S. B. Eryilmaz, D. Kuzum, R. Jeyasingh, S. B. Kim, M. BrightSky, C. Lam, and H.-S. P. Wong, "Brain-like associative learning using a nanoscale non-volatile phase change synaptic device array," Front. Neurosci., vol. 8, no. 205, 2014, DOI: 10.3389/fnins.2014.00205.

[15] Y. Zhou, H. Wu, B. Gao, W. Wu, Y. Xi, P. Yao, S. Zhang, Q. Zhang, and H. Qian, "Associative memory for image recovery with a high-performance memristor array," Adv. Funct. Mater., vol. 29, no. 30, 1900155, 2019, DOI: 10.1002/adfm.201900155.

[16] D. Kuzum, R. G. D. Jeyasingh, S. Yu, and H.-S. P Wong, "Lowenergy robust neuromorphic computation using synaptic devices," IEEE Trans. Electron Devices, vol. 59, no. 12, pp. 3489-3494, 2012, DOI: 10.1109/TED.2012.2217146.

[17] V. Milo, D. Ielmini, and E. Chicca, "Attractor networks and associative memories with STDP learning in RRAM synapses," in IEEE IEDM Tech. Dig., pp. 263-266, 2017, DOI: 10.1109/IEDM.2017.8268369.

[18] V. Milo, E. Chicca, and D. Ielmini, "Brain-inspired recurrent neural network with plastic RRAM synapses," in IEEE Int. Symp. Circuits and Systems (ISCAS), pp. 1-5, 2018, DOI: 10.1109/ISCAS.2018.8351523.

[19] N. Diederich, T. Bartsch, H. Kohlstedt, and M. Ziegler, "A memristive plasticity model of voltage-based STDP suitable for recurrent bidirectional neural networks in the hippocampus," Sci. Rep., vol. 8, no. 9367, 2018, DOI: 10.1038/s41598-018-27616-6.

[20] X.-J. Wang, "Decision making in recurrent neuronal circuits," Neuron, vol. 60, no. 2, pp. 215-234, 2008, DOI: 10.1016/j.neuron.2008.09.034.

[21] S. Kirkpatrick, C. D. Gelatt Jr, and M. P. Vecchi, "Optimization by simulated annealing," Science, vol. 220, no. 4598, pp. 671-680, 1983, DOI: $10.1126 /$ science.220.4598.671.

[22] J. J. Hopfield, "Searching for memories, Sudoku, implicit check bits, and the iterative use of not-always-correct rapid neural computation," Neural Computation, vol. 20, no. 5, pp. 1119-1164, 2008, DOI: 10.1162/neco.2007.09-06-345.

[23] F. Cai, S. Kumar, T. Van Vaerenbergh, R. Liu, C. Li, S. Yu, Q. Xia, J. J. Yang, R. Beausoleil, W. Lu, and J. P. Strachan, "Harnessing intrinsic noise in memristor Hopfield neural networks for combinatorial optimization," arXiv preprint, arXiv:1903.11194, 2019.

[24] J. H. Shin, Y. J. Jeong, M. A. Zidan, Q. Wang, and W. D. Lu, "Hardware acceleration of simulated annealing of spin glass by RRAM crossbar array," in IEEE IEDM Tech. Dig., pp. 63-66, 2018, DOI: 10.1109/IEDM.2018.8614698. 